\section{Psychotherapie und Kosten}

Der wirtschaftliche Einsatz verfügbarer Mittel ist Voraussetzung für die dauerhafte Finanzierbarkeit und damit die Erhaltung und Steigerung der Leistungsfähigkeit des Gesundheitswesens [1]. Umgekehrt ist eine leistungsfähige Wirtschaft auf die Gesundheit der diese Mittel erwirtschaftenden Bevölkerung angewiesen. Wenn also die Beurteilung ärztlicher Leistungen nach wirtschaftlichen Kriterien zur Gewohnheit werden soll, so darf sich die Sicht weder alleine auf die Kosten einengen lassen, auch wenn deren Wachstum die oberflächliche Wahrnehmung beherrscht, noch auf das individuell-medizinisch Wirksame beschränken, wie das von einem ethisch verklärten Standpunkt aus geschehen könnte, sondern es müssen beide sich gegenseitig beeinflussende Aspekte zusammen in Betracht genommen werden.

In dieser Ausgabe der Schweizerischen Ärztezeitung illustriert Heiner Lachenmeier diese Zusammenhänge am Beispiel der wirtschaftlichen Relevanz psychiatrischer Erkrankungen und ihrer Behandlungen [2]. Er kann zeigen, dass die adäquate Behandlung psychischer Erkrankungen nicht nur Kosten verursacht, sondern dass mit dem - erwiesenen medizinischen Erfolg psychotherapeutischer Massnahmen auch volkswirtschafticher Nutzen verbunden ist. Angesichts der enormen sozialen und ökonomischen Last, welche psychische Erkrankungen heute und vermutlich in Zukunft noch vermehrt darstellen, gewinnen diese Überlegungen eine besondere Bedeutung und wecken gleichzeitig die Hoffnung, dass mit besserem Verständnis und angemessenen
Behandlungsmöglichkeiten die Last und das Leid zahlreicher psychischer Erkrankungen vermindert werden können [vgl. 3].

Aus der ökonomischen Betrachtungsweise lässt sich ableiten, dass zu den Merkmalen einer guten ärztlichen Leistung auch ihr ökonomischer Nutzen zählt. Dies wird wohl niemand bestreiten können. Bei Gewöhnung an diese Denkart könnte aber davon rasch die vereinfachende Gleichung abgeleitet werden: "Eine gute Leistung ist eine wirtschaftlich sinnvolle." Und wie jede Gleichung lässt sich auch diese umkehren und man erhält: "Eine wirtschaftlich sinnvolle Leistung ist eine gute Leistung." Von diesem Satz trennt uns nur noch ein Wort von einer weiteren, aber nun falschen Aussage: "Nur eine wirtschaftlich sinnvolle Leistung ist eine gute Leistung." Ein weiterer Schritt könnte schliesslich zu einem nachgerade verheerenden, aber häufig gemachten logischen Fehlschluss führen: "Eine wirtschaftlich nicht sinnvolle Leistung ist eine schlechte ärztliche Leistung." Mit diesem kleinen Gedankenspiel soll nur eines deutlich gemacht werden: Wir Ärzte sollen uns an die ökonomische Denkart gewöhnen, aber uns gleichzeitig auch der Gefahren ihres rhetorischen Missbrauchs bewusst sein. Es ist Lachenmeier vorzüglich gelungen, diese Zusammenhänge aufzuzeigen und damit auch ein schönes Plädoyer für sein Fachgebiet zu liefern.

M. Trutmann

1 Oggier. W. Wie und was messen Ökonomen im MentalHealth-Bereich? Schweiz Ärztezeitung 2001;82(41):2180-2.

2 Lachenmeier H. Das merkwürdige Diktat der zu kleinen Schuhe. Schweiz Ärztezeitung 2002;83(7):315-9.

3 WHO. World Health Report 2001. Mental Health: New Understanding, New Hope. http://www.who.int/whr/2001/main/en/index.htm 